\title{
Ética de los negocios y la economía de la informática y la comunicación ${ }^{1}$
}

\author{
Ricardo Cuevas Moreno*
}

\section{Resumen}

Este trabajo tiene como propósito explicar la relación que existe entre la revolución de la informática y la comunicación con la ética de los negocios. El surgimiento de ambas se ubica a partir de los años 1985; éste fue uno de los supuestos que se plantearon en trabajos anteriores relacionados con el tema de la ética de los negocios (Cuevas, Moreno, 2005a, 2005b).

En la exposición se establece que dicha relación se aprehende a partir del desarrollo histórico del modo de producción capitalista y las empresas. Esto implica observar la totalidad de relaciones tecnológicas, económicas, políticas e ideológicas en el contexto de la apropiación privada capitalista de las fuerzas productivas sociales.

Este análisis conduce a comprender que la ética de los negocios o éthique de l'entreprise — según la traducción en francés_ — nace de la necesidad de gestionar la subjetividad de los individuos para las empresas nacidas de la revolución de la informática y la comunicación (Lecuyer, 1994:119).

Palabras clave: ética de los negocios, economía de la informática y las comunicaciones, tecnologías de la información y de la comunicación; administración participativa, ideología, cultura de la empresa, modo de vida capitalista, autoridad y poder.

1 Si bien en la tercera Revolución Industrial, la informática y las comunicaciones ocupan un lugar preeminente, ésta incluye la revolución en la producción de materiales, la investigación biomédica y las nuevas formas de reproducción del capital social y la vida social en su conjunto.

* Investigador de la Facultad de Comercio y Administración de Tampico de la Universidad Autónoma de Tamaulipas. Correo electrónico: rcuevasfr@yahoo.fr 


\section{INTRODUCCIÓN}

【as rápidas transformaciones tecnológicas, económicas y políticas iniciadas a Lmediados de la década de 1980 han puesto a la moral y a la ética al orden de día. El triunfo del liberalismo ha significado el primado de la empresa capitalista; la influencia de ésta alcanza el conjunto de la vida social y natural.

Esta renovación del interés por la ética y la moral es originada, por un lado, por actividades de gran alcance debido a sus repercusiones para la vida social y natural: 1) la investigación médica y biológica; y 2) la economía, la política y la administración de las organizaciones. Por otro lado, los casos de corrupción y los daños al medio ambiente - en los cuales se han visto involucradas las empresas - han puesto a la orden del día la responsabilidad de ellas y sus dirigentes. Así, en un corto tiempo (1985 a nuestros días) la ética de los negocios (Dermagne, 1996), la responsabilidad social de la empresa y el desarrollo sustentable devienen en temas integrantes de la administración cotidiana de la administración y de las ciencias de la administración.

El énfasis en la importancia de la ética y la moral para la administración concierne todas las actividades internas y externas de la organización, de tal forma que los especialistas hablan de marketing ético, ética financiera, administración moral de los recursos humanos o moral de los negocios (Boyer, 2002).

Hasta ahora los escritores de la ética de la empresa — como nosotros nombramos a los especialistas de la ética de los negocios - han tomado como un supuesto el establecimiento de la economía de la informatización ${ }^{2}$ (EI) dentro del juego de suposiciones que justifican la necesidad del tema moral o ético para la empresa y

\footnotetext{
2 La categoría economía de la informatización es tomada de Hardt y Negri (2002). La explicación de estos autores sobre el origen de la globalización integra los distintos niveles: económico mundial, soberanía, evolución de las empresas, relación entre proletariado moderno y burguesía, junto con sus correspondientes expresiones ideológicas. Se trata de una explicación que parte de la totalidad dialéctica, como piedra de toque del conocimiento. La categoría economía de la informatización sirve para diferenciar la explicación crítica propuesta por Hardt y Negri (2000) del resto de las explicaciones parciales. En este trabajo se retoma dicha perspectiva.
} 
Ética de los negocios y la economía

de la informática y la comunicación

los negocios. Sin embargo, nosotros pensamos que esa relación no es así de simple, al contrario, es fundamental para comprender el origen y contenido de esa renovación del interés de la moral para la empresa y los negocios. Este artículo tiene el objetivo de explicar esa relación.

La estructura de este trabajo es la siguiente: a partir de la perspectiva del materialismo histórico y el método de la dialéctica materialista, en una primera parte explicamos el origen de la ética de los negocios; la segunda parte aborda los cambios en la administración de la empresa provocados por la nueva infraestructura dada por la economía de la informatización; en esa misma parte ejemplificamos esas transformaciones con el caso de Francia; finalmente, presentamos las conclusiones que se desprenden de nuestro estudio.

Origen de la problemática. Existe una serie de factores comúnmente aceptados para justificar la entrada de la ética en los negocios, la cual se da en dos tiempos: primero, los cambios en el mundo actual: 1) el fin del periodo llamado por los historiadores de los "treinta gloriosos"; 2) la crisis de los valores y las instituciones como el Estado y de la familia; 3) el fin del llamado "socialismo real" y el derrumbe del Muro de Berlín; 4) la corrupción en la política y las finanzas; 5) el paso a una nueva moral hedonista; 6) la investigación biomédica (clonación y trasplantación de órganos); y 7) la globalización financiera (Daigne, 1991: 12-17).

Segundo, los efectos de esos factores sobre las empresas y los negocios, los cuales implican la moral y la ética: 1) el reconocimiento de la interrelación entre la economía, la política, la moral y los valores culturales; 2) la necesidad de la intervención de la empresa en los asuntos de la sociedad debido a los cambios en el mundo; 3) la aceptación del componente moral y ético en la toma de decisiones de los dirigentes y los administradores de las organizaciones; y 4) la existencia de nuevos espacios de autonomía y libertad para el individuo que trabaja para la empresa.

Ésos son los supuestos y al mismo tiempo las razones evocadas por los escritores de la ética de la empresa que justifican la necesidad de la investigación y recomendaciones morales para la administración de los negocios.

Los especialistas olvidan — según nosotros - las leyes de tendencia histórica de nuestra sociedad. Sin embargo, es necesario estudiar las relaciones que esos cambios guardan entre sí y sus causas con la finalidad de responder algunas cues- 
tiones capitales: ¿cuál es la relación del desarrollo del modo capitalista con la economía de la informatización?; ¿qué relación existe entre la economía de la informatización, la ética de los negocios y la administración participativa?; ¿qué tiene que ver esto con el primado del mercado y la ideología del capital?

Pensamos que las respuestas se encuentran en la historia del mundo de vida burgués, pero vista en sus contradicciones múltiples, en sus leyes de tendencia. En consecuencia, nosotros defendemos la hipótesis que es posible explicar el surgimiento de la ética de los negocios, a partir del estudio del desarrollo histórico del capitalismo y de las empresas.

En efecto, la reproducción del modo de vida burgués es la reproducción de los medios materiales, físicos y espirituales. El capital pone a su servicio todos esos medios para la obtención de la ganancia; pero también para el control y el ejercicio de la autoridad indispensable para la consecución de ese objetivo fundamental (Marx y Engels, 1982c) (Marx, 1965b). Esto desemboca en el dominio ideológico y la legitimación del estado de cosas en la sociedad y en la empresa.

\section{ORIGEN DE LA ÉTICA DE LOS NEGOCIOS}

Los especialistas del tema ético para las empresas definen en un sentido convencional; para ellos, la ética de los negocios es la disciplina que ayuda a discernir lo que es bueno, recomendable, correcto en la administración de las empresas capitalistas, desde su punto de vista ético y moral. Según Hill y Jones (2000: 57), el propósito de la ética de los negocios es suministrar herramientas para tratar la complejidad moral de las decisiones estratégicas, más que prescribir lo que es bueno o malo. Una prolongación de esta formulación englobaría toda toma de decisión y toda actividad dentro de la empresa.

Esa definición comúnmente aceptada deja de lado las transformaciones materiales sobre las cuales la ética de la empresa se origina. Así, para dar origen a la revolución en la informatización, usando la expresión de Hardt y Negri (2000), las empresas capitalistas viven importantes transformaciones en su estructura y administración. 
Ética de los negocios y la economía

de la informática y la comunicación

Uno puede comprender dichas transformaciones a través de dos explicaciones - a nuestro juicio - complementarias y excluyentes: La primera privilegia el mercado y la distribución (Chandler, 1988 y Verley, 1994). La segunda que reconoce el primado de la producción (acumulación de capital) en interrelación con la distribución y el consumo (Marx, 1965b, 1965d).

A partir de las obras de Chandler (1988), Verley (1994) y Marx (1965b) podemos establecer los periodos siguientes en el desarrollo de la empresa capitalista a partir de su estructura:

1. La manufactura o empresa tradicional o empresa preindustrial (desde la mitad del siglo XVI hasta el último tercio del siglo XVIII) y la empresa industrial moderna (1880-1930). Ésta llega a su madurez hacia 1920-1930.

2. Durante ese periodo, la empresa capitalista evoluciona de la empresa del empresario (usando la expresión de Chandler) a la empresa de administradores (usando la expresión de Marx).

3. Esa empresa pasa con la conformación del capital monopólico a la gran empresa multinacional y los conglomerados hasta 1970.

4. A partir de ese periodo asistimos a la emergencia de la empresa descentralizada en red de nuestros días. A esas empresas corresponden cambios en su infraestructura, su estructura y su organigrama conservando en su fundamento relaciones de producción capitalistas.

$\mathrm{Al}$ origen de estas estructuras se encuentra la creciente acumulación de capital (cambios cuantitativos en la masa de capital y su valor; y cambios cualitativos en la capacidad productiva del capital). La interacción de esas transformaciones con la creciente distribución y consumo de servicios y mercancías obligan a las empresas a modificar su administración y sus estructuras. Las finalidades mayores de esos procesos son la supervivencia frente a la competencia, la ganancia y la conquista del mercado.

Por consecuencia, es a partir de ese contexto que la importancia de las tecnologías de la información y la comunicación para las empresas capitalistas debe ser comprendida. Del mismo modo, debe ser comprendida la influencia de las empresas capitalistas sobre las tecnologías de la información y la comunicación; es decir, desde un doble supuesto: por un lado, la apropiación capitalista del 
conocimiento, de la tecnología, de los medios de producción, de las fuerzas de la naturaleza y de la fuerza de trabajo; por otro, las relaciones de poder, de autoridad, ideológicas y simbólicas que una tal apropiación fundan y reproducen.

Este hecho es de la más alta importancia. El primado de las comunicaciones y de la información es un supuesto sobre el cual la ética de la empresa basa su discurso; a partir de lo anterior, la ética de la empresa o ética de los negocios solicita la integración y la identificación del individuo con los valores y el proyecto de la empresa. Sin embargo, la ética de los negocios olvida las transformaciones de la composición técnica del capital y del modelo de acumulación del capital; del mismo modo, olvida los conflictos de clase y de capa de clase entre el capital y el Estado. Esos elementos son la explicación básica del primado del trabajo inmaterial y de la necesidad de la transformación de la subjetividad de la fuerza de trabajo, condition sine qua non de la economía de la informatización. Éste es el origen del renovado interés de los negocios por la moral, de la ética de los negocios y de la ideología del primado del mercado de nuestros días.

Así, para la empresa, la administración participativa proviene de esas transformaciones en la estructura económica constituida por los hombres que trabajan en ella. Esos cambios son producto, en gran medida, de las contradicciones de clase y la innovación tecnológica, aunque los especialistas de la ética de los negocios las dejan fuera del análisis a pesar de que esas contradicciones y esas innovaciones están en el contenido de la administración participativa.

Por el contrario, la administración de recursos humanos actual se refiere a otro tipo de contradicciones. Ribette en su artículo "Gérer a à temps les Ressources Humaines" establece que la administración de recursos humanos propone un nuevo modelo de gestión de contradicciones opuesto al modelo instrumental del pasado. En ese modelo, la cultura y los valores son fundamentales para la nueva administración (Simon e Joffre, 1997). La forma exterior como esta necesidad se manifiesta es a través de la noción de la cultura de la empresa que algunos autores como Mintzberg (2001) hacen equivalente con la de ideología. En otros términos, la administración participativa requiere una ideología del capital para difusión de su discurso y su ejercicio. 
Ética de los negocios y la economía

de la informática y la comunicación

\section{El paso de Dirección del Personal a la Dirección de Recursos Humanos y la mercancía fuerza de trabajo}

En la economía de la informatización, la mercancía continúa siendo el crisol de las relaciones de producción capitalistas (Marx, 1965b). A partir de los años sesenta, la economía capitalista se orienta hacia la producción de mercancías más variadas, personalizadas y de corto periodo de vida. A la producción estandarizada y a la baja de costos se sucede la producción por diferenciación y la innovación continua de productos.

La vida más corta de los productos ocasiona un cambio en los mercados y en la demanda, a los cuales las empresas deben responder rápidamente. Cuando nosotros hablamos de un agotamiento del taylorismo y del fordismo, nos referimos a la incapacidad y a la rigidez de esas formas de administración de la producción para responder a las exigencias en la revolución de las fuerzas productivas y del mercado de la tercera revolución en la industria.

Los países del capitalismo avanzado transforman esta mercancía especial — la fuerza de trabajo - con la finalidad de hacerla adecuada para la producción basada en la informática y las comunicaciones; precisamente es ahí donde la administración participativa ocupa una posición central. Entre las transformaciones y las prácticas del nuevo modelo de gestión de la empresa, la transformación de la Dirección del Personal en Dirección de Recursos Humanos (DRH) es la pieza clave.

En efecto, a partir de los años setenta, la administración de recursos humanos desde el reclutamiento, pasando por las remuneraciones y el seguimiento de carrera alcanzan un nivel de racionalización antaño inexistente. Según Floris (1996) el objetivo manifiesto es el de optimizar las capacidades del personal con la finalidad de garantizar su adaptación rápida a la ejecución de las líneas cortas de producción, de la descentralización en la toma de decisiones, de la información, en fin, del ejercicio de la participación y de la autonomía en los diferentes niveles de la estructura de la empresa (Floris, 1996: 88-89, 102-103, 106). 
En consecuencia, asegurar la adhesión de los trabajadores a la cultura y a los valores de la empresa se convierte en una actividad fundamental, incluso a partir del reclutamiento de la fuerza de trabajo y, como es evidente, durante la vida profesional de los obreros y los empleados. Es ahí donde la llamada ética de la empresa o ética de los negocios tiene su origen y su razón de ser.

Por lo tanto, la ética de la empresa esconde su verdadero origen histórico y de clase; al igual que su función dentro de la nueva administración. Por esa razón, ella es una ideología en el sentido de Marx y Engels (1982c); es decir, una falsa conciencia disfrazada de conocimiento que sirve para perpetuar las relaciones de autoridad, poder y dominio de una clase sobre otra; y de una capa social sobre otra (Cuevas, Moreno, 2005a y 2005b).

\section{La ausencia de sentido histórico}

La ética de los negocios o ética de la empresa se caracteriza por la ausencia de sentido histórico; pero en el momento en que es analizada a la luz de la historia del capitalismo y de la empresa se revela su carácter de clase. Por tal razón la empresa capitalista tiene como primera finalidad la producción de ganancia; a partir del cumplimiento de este objetivo es posible alcanzar los objetivos sociales y de sustentabilidad. Sin embargo, ninguno de esos objetivos sería posible sin el control y el consenso sobre el proletariado moderno y la sociedad civil.

Por lo anterior, la historia del surgimiento de la ética de la empresa revela su carácter de ideología del capital; en este sentido, ella es la ideología del capital para administrar mejor las empresas de la época de la informática y las comunicaciones.

Los antecedentes de la ética de los negocios remontan a los trabajos de los fundadores de la administración: Taylor (1911) y Fayol (1916), además, de los que George (2005) llama los filósofos de la administración: Sheldon (1923), E. Mayo (1933), Mooney (1931), Burnham (1941). Sin embargo, es con McGregor (1969) y Bennis (1969) que el lado humano de la empresa y la cultura se convierten en el objeto central para la administración eficaz; el impulso del tema ético para la empresa proviene de ese periodo de convulsión social al final de los años sesenta, justo en ese momento en que toma forma el sistema planificador Galbraith (1989). Es la época donde el creciente poder de las empresas transnacionales afecta 
Ética de los negocios y la economía

de la informática y la comunicación

notoriamente el destino de la economía y de la sociedad; pero es también la época donde la crisis del sistema y las respuestas —que el capital da alternativamente: la represión y la reestructuración - hacen necesaria una nueva legitimación del capital y de la empresa frente a la población mundial (Hardt y Negri, 2000).

Resulta evidente que la reestructuración y la legitimación del poder del capital no se reducen a las concesiones de protección social y de altos salarios; la legitimación cubre los diferentes espacios de la vida social, política e ideológica; por eso nuestra explicación del origen de la ética de los negocios cubre todos esos distintos niveles de análisis, pues es en ese terreno donde el tema moral para la administración de la empresa y la ética de la empresa encuentra su origen.

\section{El caso de Francia}

Los factores que hemos señalado explican el paso hacia una nueva etapa de la producción y de la soberanía capitalistas; asimismo, se repiten en la particularidad del capitalismo francés. En efecto, a partir de los años sesenta, las empresas francesas vivieron modificaciones en su estructura y en su administración para responder, por un lado, a las nuevas exigencias de la acumulación y la distribución en masa; y, por el otro, a la fuerza combativa de los trabajadores (Hardt y Negri, 2000).

De igual modo, la sociedad francesa vivió las modificaciones en las relaciones entre el Estado, los patrones y la sindicatos; además, se planteó una recomposición del proletariado y del tránsito a la economía de la informatización, cuyas consecuencias son el primado de los servicios y el trabajo inmaterial sobre el resto de la economía. La ideología del capital y la administración participativa para la administración de la empresa y la gestión de la vida social a escala mundial cristalizan ese proceso.

Si bien es cierto que las raíces de ese proceso se encuentran en el nacimiento de la empresa moderna, el momento del surgimiento hacia el nuevo paradigma sucede durante el periodo que va de 1980 a 1995. Floris (1996) trata de demostrar que esos rápidos cambios corresponden a las necesidades de la transformación simbólica del capital francés y de la legitimación del capital y de la empresa en el espacio público. Una de las hipótesis importantes de ese autor se centra en la 
comunicación de empresa - elemento integrante de la administración participativa - , la cual contribuye a la construcción del nuevo modelo de legitimación de la gran empresa capitalista y a una nueva relación entre los administradores y los asalariados (Floris, 1996: 51).

Floris, que no es ni economista, ni filósofo, ni administrador, es uno de los escasos espíritus críticos que se encuentran en la ética de los negocios. Él pone en relieve dos cosas curiosamente ignoradas por los especialistas del tema: por una parte, la ideología del management permite la reproducción de las relaciones de legitimidad, de dominación de la administración moderna y de la reproducción de la inigualdad en el espacio social; por otra, la necesidad de recurrir a la evolución histórica del capitalismo y la sociedad francesa en la explicación del surgimiento de lo que Floris (1996) nombra la ideología del management o ideología de la administración.

\section{La informatización y el proletariado}

La entrada de Francia en la economía de la informatización ocurre después de los EUA y aproximadamente al mismo tiempo del resto de los países de Europa. En la economía de la informatización, Francia se encuentra a la zaga de EUA y de Japón, quienes son los líderes mundiales; en los inicios del nuevo siglo, Francia se sitúa al mismo nivel que el Reino Unido, detrás de Alemania, pero antes de Italia.

Según el Institut de la Statisque et des Études Économiques (INSEE) 3 (2000), la economía de la informatización es difundida gracias a su alta productividad y a la baja considerable y continua del precio de las Nuevas Tecnologías de la Información y de la Comunicación (NTIC). Eso permite su consumo masivo de parte de las empresas y los hogares. Los productos más consumidos son internet y el teléfono celular. Bajo la clasificación NTIC la contabilidad francesa reagrupa una serie de actividades diversas: la informática, la telecomunicación y la electrónica, la fabricación de materiales, de aparatos y máquinas. El punto importante de Francia es la producción de material de telecomunicaciones mientras que los servicios en telecomunicaciones están a la baja.

\footnotetext{
${ }^{3}$ Instituto de Estadística y de Estudios Económicos (INSSE) de Francia.
} 
Ética de los negocios y la economía

de la informática y la comunicación

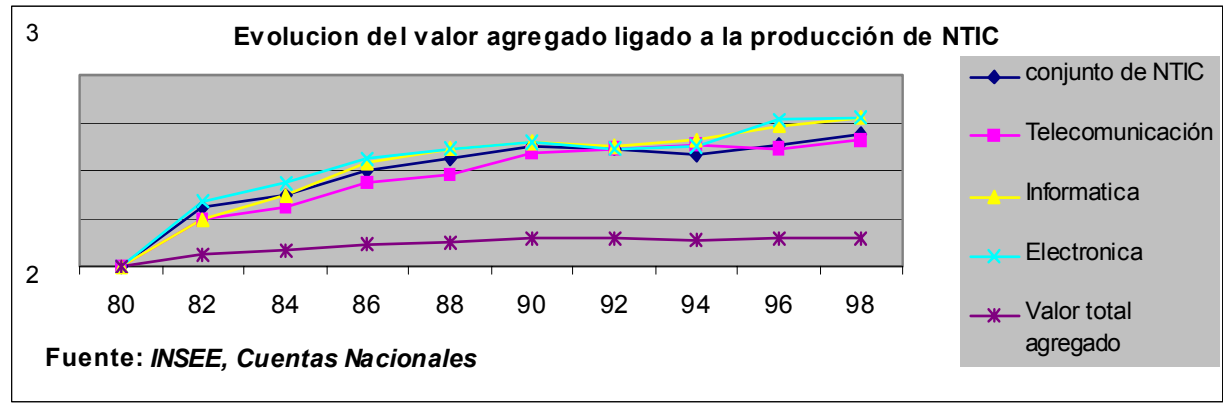

El desarrollo de la NTIC da una idea del progreso del tránsito hacia la economía de la informatización de la economía francesa, a pesar de que los servicios financieros se encuentran ausentes de la clasificación contenida en la gráfica. El crecimiento de las NTIC y su dinamismo ocupan una parte cada vez más importante en esa economía.

La rama de "actividades informáticas" ha experimentado un crecimiento continuo, después de una ligera desaceleración durante los años ochenta. Su crecimiento proviene de las actividades de servicios, cuyo valor agregado es diez veces más elevado en 1998 que en 1980. Al origen del dinamismo de los servicios de informática se encuentra la difusión de los materiales, el cual repercute sobre la demanda de servicios conexos de las empresas y los hogares (INSEE, 2000:87-104).

Durante el periodo de 1967-1997 las inversiones en materiales informáticos se multiplican por veinte en valor; en el mismo periodo, el crecimiento del capital informático medio anual fue de $31 \%$ y la de equipos $4,2 \%$. La contribución al PIB del capital informático medio anual fue de $0,10 \%$ para un crecimiento del PIB de $2,9 \%$ medio anual. Según el INSEE, el peso del capital de informática es débil en relación con el PIB, él ha contribuido de manera notable al crecimiento económico general (INSEE, 2000:127-132).

Por otro lado, los efectivos empleados de la NTIC aumentaron alrededor del 20\% en diez años (1980-1998). Su parte dentro del empleo total pasa de 2,5\% a 2,9\%. El aumento de los efectivos de los servicios se degradó en un 8\% entre 1991 y 1994 para remontar vivamente hasta 1998, recuperando su nivel precedente. En 1998 las NTIC ocupan 658 mil personas, es decir, 2,9\% de los efectivos 
empleados. Según el INSEE, el progreso en el número de efectivos se aplica por el dinamismo de servicios informáticos. Hacia 1998 esa rama ocupó 230 mil personas; de 1980 a 1998 esa rama creó un total de 50 mil empleos.

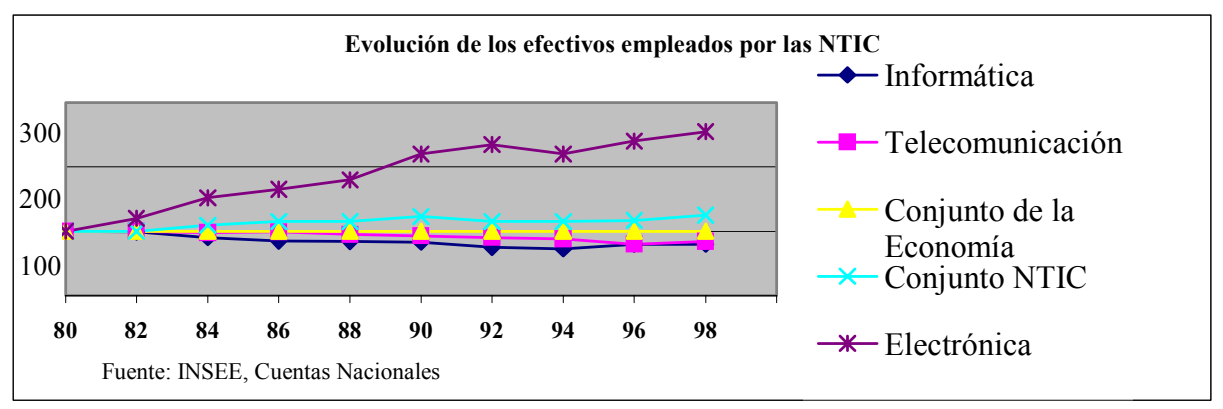

\section{Recomposición del proletariado}

Existe una base material sobre la cual ese proceso es posible - que se constituye, en primer lugar, por las redes formadas por las empresas en la investigación científica, especialmente en la biotecnología, la información y la comunicación, las cuales funcionan retomando en modelo japonés de administración de la empresa; en segundo lugar, el sindicalismo militante de los años sesenta construyó una red de información y de comunicación (los comités y delegados, los diarios y las revistas), los cuales constituyen hasta nuestros días los vehículos de difusión de la ideología al interior de la empresa; en tercer lugar, de esa época datan las declaraciones de la necesidad de reestructurar la empresa y modificar la mentalidad del patronato francés, centralizador y autoritario. En fin, en esa época nace el proceso de recomposición del proletariado y la emergencia de los empleados, mandos medios y superiores necesarios para la economía de la informatización.

En ese sentido, la entrada masiva a las universidades, que es un factor explicativo primordial del movimiento de los años 1968 , también provee a largo plazo los empleados, los mandos medios y superiores para la transformación estructural de la economía francesa. 
Ética de los negocios y la economía

de la informática y la comunicación

Según Floris (1996), los cuadros especializados en administración y en comunicación surgen de las grandes escuelas francesas (ENA, Mines, Polytechnique, HEC). Esos mandos de alto nivel de las grandes instituciones navegan entre el sector público, el sector privado y la política dando un trazo particular al capitalismo francés. Esos cuadros juegan un rol no despreciable en la difusión de la administración participativa y su ideología. Los otros factores de esta difusión son la participación del Estado y los medios masivos de comunicación.

En fin, nosotros subrayamos una idea fundamental establecida por Marx (1965f: 164), (Marx, 1965b: 859-999): el capital se apropia de todas las fuerzas productivas (objetivas y subjetivas) de la sociedad y las pone al servicio de la producción de ganancia. En suma, solamente de esa manera es posible explicar la adopción de la nueva administración, su ideología a partir de los años 1980 y su generalización en las organizaciones hacia 1996.

\section{LA ADMINISTRACIÓN DE LA EMPRESA Y LA ECONOMÍA DE LA INFORMATIZACIÓN}

La economía de la informatización requiere estructuras flexibles capaces de adaptarse a los diferentes medios y de responder rápidamente a los cambios de la producción y del mercado, pues las grandes empresas deben tener la capacidad de gestionar las diferencias internas en los distintos países de origen y las diferencias multiculturales en el extranjero. La administración moderna llama a ese objeto administración intercultural.

Entonces, el rol del mánager o de la tecnoestructura es provocar la sinergia necesaria para que esa nueva estructura sea eficaz y alcance sus objetivos. De ello se desprende que el mánager busca optimizar las energías, la creatividad, el libre juego de las potencialidades del proletariado moderno. Esas tareas tienen lugar en cada punto del organismo descentralizado. De ahí proviene la apariencia de autonomía, de igualdad y de democracia que la ideología del capital emplea para ocultar el hecho que todos los puntos son administrados y conectados al centro de autoridad.

Para la empresa y la administración esos cambios se expresan, por una parte, en la sustitución del modelo inspirado en la teoría de F. Taylor (1986) y H. Ford por el modelo de Toyota, y, por otra, en la producción inmaterial de servicios (financie- 
ros, culturales y de educación). Según Hardt y Negri (2000: 303-307, 319-333, 359363) al final de la década de 1960, los modelos tayloriano y fondista sobrepujaron la racionalización de la producción al extremo; en aquella época, esos modelos ponen en evidencia su incapacidad para continuar dinamizando las fuerzas productivas y controlar la subjetividad proletaria producida por ellos mismos.

En la práctica, la descentralización de la producción significa una reestructuración física del capital y su administración. En la actualidad el modelo organizacional de la producción en cadena de montaje es sustituido por el de red de comunicación. Así, el modelo toyotista se funda sobre la inversión de la estructura entre la producción y el consumo fordista. El toyotismo reduce la diferencia entre el tiempo de producción y el tiempo de realización del producto. La aplicación ideal de ese modelo es el de crear el producto solamente después de haber sido demandado. Las consecuencias para la reducción del tiempo de trabajo social necesario son evidentes porque inciden en el punto crucial del ciclo del capital: la realización de plusvalía.

Los efectos de la nueva estructura, del nuevo modelo de acumulación de capital y de la administración, hacen de la empresa una entidad aún más poderosa. La prueba de ello no son sólo el maná de riqueza, la plétora de capital producido, sino el poder ejercido de las empresas sobre el mundo, sobre todo a partir del derrumbe del llamado "socialismo real". El peso de las empresas en la producción mundial de riqueza es notable, 29 multinacionales se encuentran entre las cien primeras entidades económicas mundiales (Novethic. fr, 24/septiembre/2004).

Estos hechos han puesto la moral al orden del día para las empresas en tres ejes: responsabilidad (económica y social), derechos del hombre y medio ambiente. Hemos visto que en el corazón de esos fenómenos se encuentran la revolución en la informática y las comunicaciones bajo su apropiación privada capitalista.

\section{¿Son las prácticas de la informatización las que demandan la ética?}

La pertinencia de esta pregunta proviene de la necesidad de no confundir las causas con los motivos, es decir, los contenidos con sus formas exteriores. En efecto, la implantación de la economía de la informatización posibilita la transformación del mundo de una forma nueva. 
Ética de los negocios y la economía

de la informática y la comunicación

El ejercicio del poder sobre la naturaleza y la sociedad es uno de los fundamentos éticos del principio de responsabilidad (Jonas, 2000). Así, el tratamiento de la información financiera, la clonación de seres vivos, la trasplantación de órganos, la educación y la difusión mundial de la cultura son expresión de una mayor potencia de la capacidad productiva social del trabajo de una calidad nueva.

En su ejercicio todas esas prácticas implican el comportamiento moral de los individuos. Implican el comportamiento moral de acuerdo con los valores establecidos por la sociedad burguesa. Si bien que la ley moral se cumple o se infringe, es innegable la presencia de la moral en esas actividades y sus efectos; como también es innegable la necesidad de la reflexión de la ética sobre los mismos.

El control capitalista privado de la nueva infraestructura de la informática y las comunicaciones da un poder enorme a las empresas y los gobiernos que se han puesto en la vanguardia de esa revolución. Dicho poder no sólo se ejerce sobre la naturaleza, lo cual implica ya una responsabilidad, honestidad y transparencia y, en consecuencia, la moral. Decir que el poder se ejerce de unos pueblos sobre otros — aunque si bien es cierto - olvidamos que ese poder se ejerce de los hombres sobre los hombres mismos, mediante las empresas, los Estados nacionales y las armas.

El eslogan, hoy popular, que dice "Este siglo será ético o no lo será" es la forma exterior y exagerada de la necesidad de la moral y la ética que las actividades en la producción y la circulación basadas en la economía de la informatización ponen en relieve.

Aclaremos, no que la ética y la moral hayan sido menos necesarias antes de 1985, que ahora, si no que su renovado interés para la administración de las empresas tiene como fundamento esa nueva infraestructura dada por el conocimiento en la informática y las comunicaciones. Las nuevas prácticas que esa nueva infraestructura hace posible (control de la subjetividad, trabajo inmaterial, investigación biomédica, trasplantación de órganos y especulación financiera) hacen necesario un límite y un criterio de verdad.

En efecto, la moral establece los límites de las pulsiones, los deseos y el interés egoístas; asimismo, ella es un criterio de verdad en relación con lo que se considera bueno, positivo, correcto y recomendable. La administración moral de la empresa es la forma exterior que toma la necesidad de poner al hombre y a su praxis en el centro del análisis. 
La necesidad de la moral y la ética, originada por la transformación del mundo con las Nuevas Tecnologías de la Información y de la Comunicación (NTIC), es un estado del organismo social; es una reacción para establecer un coto a los deseos de ganancia y poder, a los individuos, a las empresas y a los gobiernos; además, es un criterio de verdad sancionado por los hechos, es decir, por las condiciones de vida de la masa. No son las prácticas de la economía de la informatización las que demandan la moral y la ética, sino son los hombres y las mujeres quienes los realizan y esas prácticas son las que necesitan la ética y la moral.

De lo anterior se deriva que en el mundo de los negocios no son sólo las empresas o la sociedad las que demandan la ética y la moral, sino son los hombres los que realizan su praxis dentro de la empresa o fuera de ella; ellos demandan y requieren el ejercicio de la moral y la comprensión de la ética; y lo hacen porque viven la violación de sus derechos universales o sufren la degradación de su hábitat y su nivel de vida.

Considerar a los negocios como una entidad aparte en la cual la moral y la ética no tienen nada que ver, sino de manera exterior es un grave error que imposibilita comprender la relación orgánica entre ética, moral y negocios; y en un sentido más amplio la relación entre ética, economía y administración. Al contrario, la praxis como totalidad de relaciones permite ver la diferencia de esos mundos y su relación orgánica.

Sin embargo, esa ruptura entre el mundo de los negocios, la moral y la ética se explica por el primado del interés, el egoísmo y el despotismo en los primeros. Es de esta forma contradictoria cómo lo humano se expresa en el mundo de los negocios, aunque dentro de los mismos hay gente progresista guiada por los más altos valores morales.

Por otro lado, la ruptura entre economía, administración y ética se explica por el primado de la razón racionalista o razón burguesa, que ha dividido al mundo en dos: por un lado, el mundo del cálculo, de la medida, de lo que es mesurable y por tanto racional; por el otro, el mundo de las emociones, los sentimientos y las pasiones, no mesurables y, por tanto, fuera de la racionalidad (Kosik, 1970). La crítica de la razón racionalista nos conduce a comprender que esos mundos son en realidad una totalidad; los hombres en su vida práctica reproducen esa totalidad donde confluyen esos dos mundos. 
Ética de los negocios y la economía

de la informática y la comunicación

Así, en la empresa y los negocios, la ética y moral están presentes como parte de su ser y constituyen un todo. La demanda de una administración moral para las empresas y los negocios es la forma exterior cómo se presenta la reacción contra la ruptura provocada por la simple búsqueda del provecho. Es la forma como lo humano en todas sus dimensiones busca restaurarse frente al trabajo alienado.

\section{La ética de los negocios de la época de la informatización y el destino del hombre}

Todo lo anterior nos conduce a una pregunta crucial olvidada por los especialistas de la ética de los negocios ¿cuál es el destino del hombre de esta época de la informatización?

Expliquemos, para los escritores de la ética de los negocios no es el "hombre", sino el "individuo" o el "actor" el punto clave del análisis. Así, el actor reproduce el sistema eligiendo las estrategias pertinentes — de acuerdo con la racionalidad limitada - que le permitan obtener el mejor provecho dentro del sistema del cual él forma parte (Archier y Sérieyx, 1984). Por ejemplo, el individuo debe aprender y practicar el comportamiento moral dictado por los códigos éticos porque de esa manera identificará su proyecto personal con el proyecto de la empresa. Esta adhesión promete el florecimiento personal; sin embargo, el individuo o el actor, dentro de la empresa, actúan como entidad parcial, autónoma pero subordinada a un centro estratégico. La revolución de la informatización no ha abolido el trabajo alienado, o la enajenación de trabajo capitalista.

Al contrario, la nueva infraestructura posibilita la enajenación y el control de la subjetividad: el primado del trabajo inmaterial, (el tele-trabajo) es un ejemplo de este nuevo tipo de trabajo abstracto. De esta forma, la autonomía y la libertad del trabajador — con las cuales se postula la abolición de conflictos de clase dentro de la empresa y la abolición de las clases sociales en los países desarrollados - son del todo aparentes. Todos los trabajos son administrados en los distintos centros estratégicos ligados por la red.

El hecho fundamental es que el capital sigue determinando el destino de la masa de empleados y trabajadores. La ética no sólo nos dice cuáles son las leyes del comportamiento moral, los valores morales y la conciencia moral de los hombres, 
sino también nos explica el sentido de ese comportamiento. Este sentido implica un destino, un lugar hacia el cual uno se dirige, un lugar de llegada y, por tanto, una posición, un sitio dentro del modo de vida del organismo social.

Lo anterior significa un cambio de paradigma mundial; se trata de un cambio económico, político y social: la razón más importante es que el mercado mundial aparece como la pieza central en el dispositivo del control de la producción. El mercado es también la pieza central del control y de la legitimación de la sociedad disciplinaria, entonces, por el mercado mundial actual las fronteras del Estado-nación aparecen como obstáculos, los cuales son superados gracias al poder descentralizado en red de las empresas y los organismos sociales y políticos. Gracias al mercado mundial, las formas de producción y de vida disciplinarias del capitalismo son difundidas a lo largo de su historia. La realización del mercado — de su estructura — puede ser concebida como la forma emblemática del poder imperial; la realización del modo de vida capitalista, de su poder, de su disciplina y de su legitimidad (Hardt y Negri, 2000).

La acumulación creciente del capital significa la socialización creciente de la producción: la generalización del mercado mundial es la generalización de la lucha de clases y de la crisis a escala mundial. La dinámica del modo de vida capitalista pone frente a frente a la masa de proletarios desposeídos y la apropiación privada de la riqueza social de la clase capitalista.

Por lo tanto, la realización del mercado mundial actual cumple perfectamente esta tesis sostenida por Marx (1965b), salvo que se trata de la movilidad mundial de la fuerza de trabajo y de un nuevo proletariado, es decir, del proletariado moderno o proletariado de la economía de la informatización. Ahí se cumple todavía la tendencia a someter a la población al trabajo salariado; así, el primado del proletariado de la fábrica del siglo XIX es sustituido por el primado del trabajo salariado en los servicios, lo que da como resultado que la multitud de desposeídos y el capital se encuentran cara a cara; en ese sentido, la realización del mercado mundial es la realización de esa contradicción.

El capital como relación de producción, es decir como relación entre hombres mediante las cosas, toma forma en la organización llamada empresa capitalista; además, el capital y la empresa siguen determinado el destino del hombre de la época actual, pero lo hacen sobre una nueva infraestructura la cual posibilita el 
Ética de los negocios y la economía

de la informática y la comunicación

control del ejercicio físico, de la subjetividad y sus resultados. Lo anterior sucede en todas las empresas y organizaciones susceptibles de adoptar las nuevas tecnologías de la información y de la comunicación (NTIC) en su administración. En suma, el destino del hombre es servir al capital, pero esta vez en cuerpo y alma asumiendo los valores de su organización.

La administración moral de los negocios contiene un componente ideológico sin los cuales ella no puede ser explicada, ni tiene razón de existir; esto es, tiene relaciones de propiedad, de autoridad y de poder que marcan el destino del hombre de nuestros días a escala mundial.

\section{CONCLUSIONES}

1. El surgimiento y función de la ética de los negocios puede ser explicada a partir de la emergencia de la revolución de la informática y las comunicaciones. Ambas son la creación del desarrollo del modo de vida burgués considerado en sus relaciones económicas, políticas, sociales e ideológicas. En otros términos, ambas son creación de los hombres que viven en ese modo de vida.

2. La tercera revolución en la informática y las comunicaciones, al hacerse inmanente a los procesos de la producción y la distribución social, crea la base material de un nuevo modelo de acumulación. De ahí proviene la necesidad de una nueva forma de administración. La administración participativa, la comunicación corporativa y la calidad total representan unas tantas formas de esta nueva administración.

3. Un rasgo importante de esta nueva administración moral es la gestión de la subjetividad, si bien la nueva infraestructura la posibilita, ésta no sería posible sin la legitimación y aceptación por parte de los hombres que trabajan en las empresas y las organizaciones. En este sentido la transformación de la Dirección de Personal en Dirección de Recursos Humanos es clave.

4. Es bajo estas ideas cómo se explica el origen de la ética de los negocios y su razón de ser. Ella es la ideología del capital para mejor administrar la empresa de la economía de la informatización. 
5. La demanda de una administración moral de las empresas y las organizaciones es del todo pertinente. La enseñanza que podemos inferir de su problemática, que es formulada por los autores de la ética de los negocios, es el hecho de poner en el corazón de los negocios al hombre que trabaja en la empresa capitalista, aunque en un sentido diferente al que se sostiene en este trabajo, pues no es el hombre sino el individuo o el actor el centro de atención.

6. Por el contrario, la ética de los negocios y las empresas no responden cómo los hombres de manera práctica pueden alcanzar el florecimiento personal bajo el primado del trabajo enajenando.

7. Los procesos señalados ocurren principalmente en los países llamados desarrollados; nos resta por explicar en nuestro programa de investigación cómo este proceso ocurre en México.

\section{BIBLIOGRAFÍA}

ARCHIER, G. et Sérieyx, H. ; L'entreprise du $3^{e}$ type, Editions Seuil, Paris, 1984.

BERGMANN, A. ; "Ethique et gestion", en Y. Simon et P. Joffre (sous la direction); Encyclopédie de Gestion, 2e édition, Economique, T. II, Paris, 1997, p. 1239.

BLANCHARD, K. et Peale N.V. ; Ethique et management, Réussir en restant intègre, Traduction par S. Marnat, Les Editions d'Organisation, Paris, 1988.

BOUILLOUD, J-P et Lecuyer, B-P ; L'invention de la gestion. Histoire et pratiques, L'Harmattan, Paris, 1994.

BOYER, A. et al. ; L'impossible éthique des entreprises, Éditions d'Organisation, Paris, 2002.

CARON, F. ; Les deux révolutions industrielles $d u X X^{\circ}$ siècle, Editions Albin Michel, Paris, 1997. 
Ética de los negocios y la economía

de la informática y la comunicación

CHANDLER, Jr. Alfred D ; La main visible des managers, Traduction de l'américain par Langer, F ; éditeur Economique, Paris, 1988 (1977).

CHARREAUX, G. ; "Gouvernement de l'entreprise", en Y. Simon et P. Joffre, (sous la direction) ; Encyclopédie de Gestion, $2^{\mathrm{e}}$ édition, Economique, T. II, Paris, 1997, p, 1652.

CLAUDE, J-F ; L'éthique au service du management, Les Éditions LIAISONS, Paris, 1998.

COHEN, E. ; "Epistémologie de la gestion", en Y. Simon et P. Joffre (sous la direction) ; Encyclopédie de Gestion, $2^{\mathrm{e}}$ édition, Economique, T. I, Paris, 1997, p, 1158.

CUEVAS MORENO, R. ; "La construction de l'éthique des affaires. Première partie. Les formes à partir de l'adoption de l'éthique", Contaduría y Administración, Facultad de Contaduría y Administración, UNAM, No. 216, México, mayo-agosto, 2005a.

; "La construction de l'éthique de l'entreprise ou l'éthique des affaires. Seconde partie. Les formes à partir de l'adoption de l'économie", Contaduría y Administración, Facultad de Contaduría y Administración, UNAM, No. 217, México, septiembre-diciembre, 2005 b.

DAIGNE, J-F ; L'éthique financière, PUF collection que sais-je ? numéro 2604, Paris, 1991.

DE FEUILHADE DE CHAUVIN, Tanneguy ; Ethique et pouvoir dans l'entreprise, ESF éditeur, Paris, 1991.

DE LA BRUSLERIE, H. (coordonnateur) ; Éthique, Déontologie et Gestion de l'Entreprise, éditeur Economica collection Gestion, Paris, 1992.

DERMAGNE, J. ; Révolution chez les patrons ? L'entreprise citoyenne, Bayard Editions, Paris, 1996. 
DESREUMAUX, A ; "Structures de l'entreprise", en Y. Simon et P. Joffre (sous la direction) ; Encyclopédie de Gestion, Economique, 2e édition, T. III, Paris, 1997, p, 3147.

ETCHEGOYEN, A. ; La valse des Ethiques, Editions François Boudin, Paris, 1991.

FABER, E. ; Main basse sur la Cité. Ethique et entreprise, éditeur Hachette, France, 1992.

FALISE, M. et Regnier, J ; Repères pour une Ethique d'Entreprise, éditeur Centre d'Ethique Contemporaine, France, 1992.

FARCY, H. (présentateur) ; Vers une morale des affaires? L'Ethique du Responsable commercial, Institut catholique de Paris. Action populaire. Bibliothèque de la Recherche Sociale. SPES, Paris, 1969 (Cas concrets introduit par FARCY, H.).

FLORIS, B. ; La communication managériale. La modernisation symbolique des entreprises, Grenoble, PUG, Francia, 1996.

GALBRAITH, J. K. ; Le nouvel Etat industriel, Gallimard, collection TEL, Traduit de l'anglais par Crémieux-Brilhac, J-L et Le Nan, M, Paris, 1989, (1967).

GELINIER, O. ; L'éthique des affaires. Halte à la dérive, Editions du Seuil, Paris, 1991.

GEORGE, S, y Álvarez, L. ; Historia del pensamiento administrativo, Pearson, Prentice Hall, Traducido del inglés, México, 2005 (1974).

INSEE ; L'économie française. Rapport sur les comptes de la Nation de 1999, Librairie Générale Française, INSEE, Paris, 2000.

HARDT, M. et A. Negri; Empire, Exils Editeur, traduit de l'américain par Canal, D-A, Paris, 2000. 
Ética de los negocios y la economía

de la informática y la comunicación

HILL, C. y Jones, G.; Administración estratégica un enfoque integrado, McGrawHill, Colombia, 2000 (1996).

JONAS, H. ; Le principe de responsabilité, Une éthique pour la civilisation technologique, Flammarion, Paris, 2000 (1979).

KOSIK, K. ; La dialectique du concret, Traduit de l'allemand par Dangeville, R., éditeur Maspero, Paris, 1970.

LALANDE, A. ; Vocabulaire technique et Critique de la Philosophie, PUF, $17^{\mathrm{e}}$ édition, Paris, 1991 (1926).

LIPOVETSKY, G. ; Le crépuscule du devoir. L'éthique indolore des nouveaux temps démocratiques, Éditions Gallimard, NRF essais, Paris, 1992.

MCGREGOR, D. ; La dimension humaine de l'entreprise, México, GauthierVillars, 1960.

MARCUSE, H. ; L'homme unidimensionnel. Essai sur l'idéologie de la société industrielle avancée, Traduit de l'anglais par M. Wittig et l'auteur, 1968 Editions Minuit, 1968 (1964).

MARX, K. ; Manuscrits parisiens, Bibliothèque de la Pléiade, Euvres III Philosophie, édition établie, présentée et annotée par Maximilien Rubel, NRF Gallimard, Paris, 1968a (1844).

; Le Capital, livre I, Bibliothèque de la Pléiade, Euvres I Economie, édition établie, présentée et annotée par Maximilien Rubel, NRF Gallimard, Paris, 1965b (1867).

- et Engels, F.; L'Idéologie allemande, Bibliothèque de la Pléiade, Euvres III Philosophie, édition établie, présentée et annotée par Maximilien Rubel. NRF Gallimard, Paris, 1982c (1846).

; Contribution à la critique de l'économie politique, Bibliothèque de la Pléiade. CEuvres I Economie, édition établie, présentée et annotée par Maximilien Rubel. NRF Gallimard, Paris, 1965d (1859). 
et Engels, F. ; Le manifeste communiste, Bibliothèque de la Pléiade, Euvres I Economie, édition établie, présentée et annotée par Maximilien Rubel. NRF Gallimard, Paris, 1965f (1848).

MERCIER, S. ; L'éthique dans les entreprises, La Découverte, Collection Repères, Paris, 1999.

MINTZBERG, H. ; Le pouvoir dans les organisations, Les Editions de l'Organisation, Traduit de l'américain par P. Sager, Paris, 1986 (1983).

- Le management, voyage au centre des organisations, Editions d'Organisation, traduit par Behar, J-M, quatrième édition, Paris, 2001 (1989).

NAUDET, J-Y ; (dirigée), Ethique des affaires : de l'éthique de l'entrepreneur au droit des affaires, Collection Ethique et Déontologie, Editeur Librairie de l'Université d'Aix-en-Provence, 1997.

ORSONI, Jacques ; "L'enseignant de gestion face à la morale d'entreprise", Revue française de gestion, $\mathrm{n}^{\circ}$ 74, juin, 1989, p, 114.

PETERS, T. et Waterman, R. ; Le prix de l'excellence. Les secrets des meilleures entreprises, InterEditions, Traduit de l'américain par M. Garene et C. Pommier, Paris, 1983 (1982).

REITTER, R ; "Culture et identité", en Y. Simon et P. Joffre (sous la direction) ; Encyclopédie de Gestion, Economique, 2e édition, T. I, Paris, 1997, p, 878.

SALOMON, R.C. et Hanson, K.R ; La morale en affaires clé de la réussite, Éd. Organisation, Paris, 1989.

SIMON, Y. et P. Joffre (sous la direction) ; Encyclopédie de Gestion, $2^{\mathrm{e}}$ édition, Economique, T. I, II, III, Paris, 1997.

TAYLOR, W. F., Los principios del management científico, Traducción Alicia Arrufat, quinta edición, Ediciones Orbis, España, 1986 (1911). 
Ética de los negocios y la economía

de la informática y la comunicación

VERLEY, P. ; Entreprises et entrepreneurs du XVIII siècle au début du XX siècle, éditeur Hachette, Paris, 1994.

www.novethic.fr (24/septiembre/2004) Repères. La Responsabilité Sociale des Entreprises. G 